\title{
326 Why tailored information and communication technologies for motor rehabilitation?
}

\author{
Gabriela Postolache, Raul Oliveira \\ Instituto de Telecomunicações, Lisbon, Portugal \\ Faculdade de Motricidade Humana, Lisbon, Portugal
}

\section{Introduction}

Information and communication technologies (ICT) have proven to be valuable resource for preventive, predictive, personalized and participatory health care. Many studies on ICT in the area of motor rehabilitation have also shown benefits for patients and care providers. However, a few empirical studies that highlight the diffusion level and the patients' awareness and perspectives on ICT for motor rehabilitation were published. We investigate the patients' perspectives on ICT for physiotherapy in order to produce actionable knowledge for development of tailored mobile technologies for motor rehabilitation monitoring.

\section{Methods}

Based on data from literature review, workshops, interviews and secondary research a structured questionnaire (42 questions, 5 point Likert scale) was developed. The questionnaire was administered with support of other person (a psychologist) or self-administered in clinic' space or online.

\section{Results of Survey}

Participants:

- 367 patients receiving physiotherapy in Portugal

aged between 18 to 87 years

138 patients age $>65 y$ (average age: $73.4 \pm 5.3$ years)
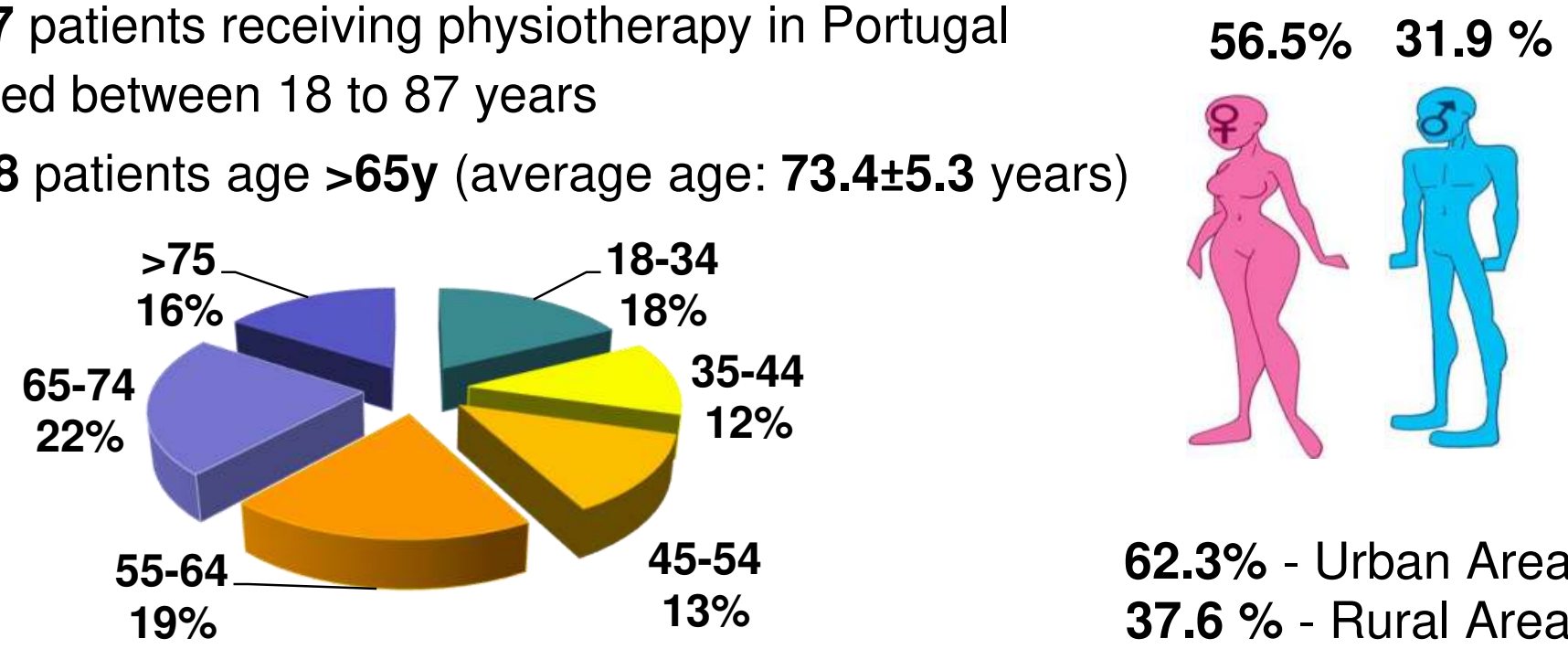

62.3\% - Urban Area $37.6 \%$ - Rural Area

At the questions 'what technologies you have or had' only a few participants responded as having devices for movements monitoring or activity tracking. Rural elderly reported having mainly television and cell phone. These data is in line with results from a recent Accenture 2017 report that showed that apps, wearable, and social media use to manage health was less in population with age $65-74$ years (16\%) and more pronounced in individuals with age 18-34 years $(48 \%)$.

\section{Q1. What importance they give for the existence of ICT that allow the following functionalities associated with Physiotherapy}

Q1.1. online access to health data:

Q1.2 health data displayed in images easy to understand;

Q1.3 better communication with their therapists;

Q1.4 communication and sharing of information between therapists and other health professionals;

Q1.5 frequent recording of health information;

Q1.6 online appointments;

Q1.7 alerts and notification.

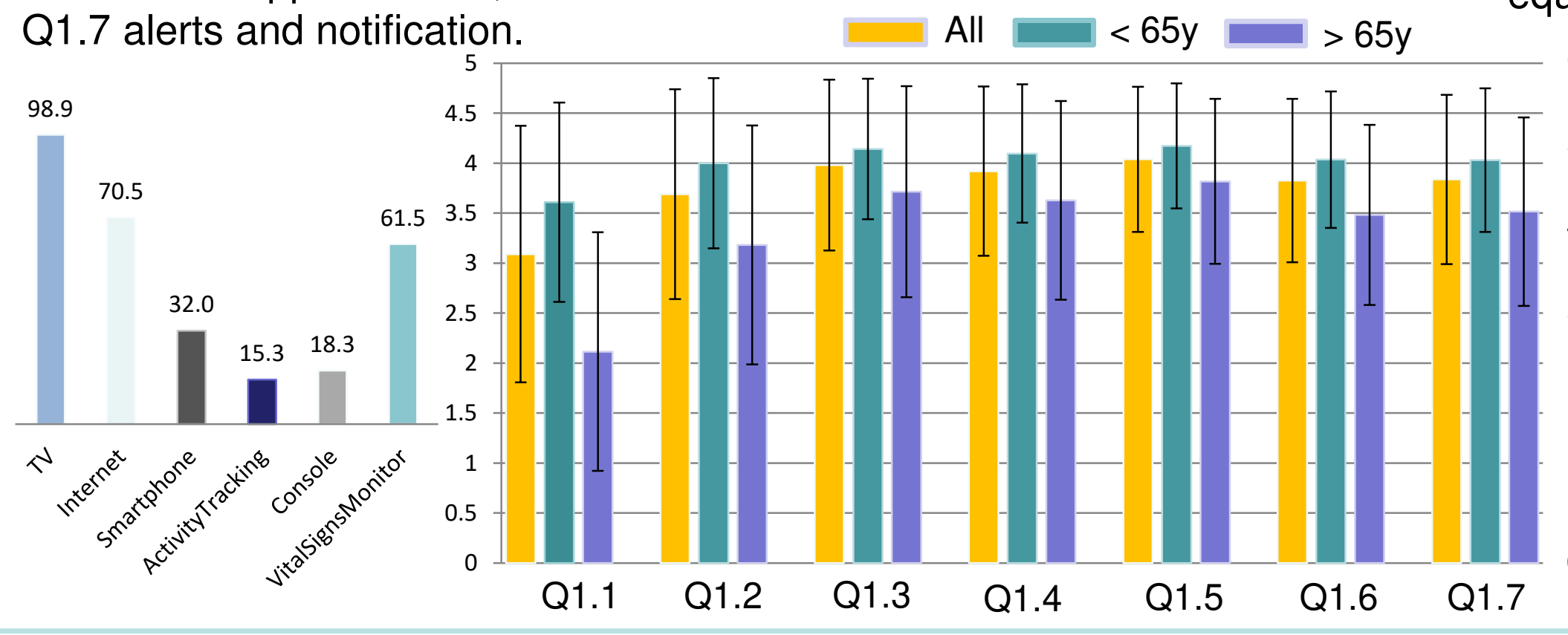

The patients considered more important the existence of ICT for physiotherapy that allow better communication with their therapists (mean $\pm S D$ : $4.0 \pm 0.8)$, communication and sharing of information between therapists and other health professionals $(3.9 \pm 0.8)$, or frequent recording of health information $(4.0 \pm 0.7)$ than update on health information (2.8 \pm 1.4$)$, online access to health information (3.1 \pm 1.3$)$, health data displayed in images easy to understand $(3.7 \pm 1.0)$, online appointments $(3.8 \pm 0.8)$ or alerts and notifications $(3.8 \pm 0.8)$, being elderly population less convinced on importance of the questioned ICT functionalities. Lower level of agreement was registered for statements related to the capability of ICT: to guarantee the security and privacy of data $(3.6 \pm 1.1)$; to allow in home training for motor rehabilitation $(3.5 \pm 1.1)$. Elderly patients perceived as less affordable these technologies (i.e., are expensive: $4.5 \pm 0.8$ versus $4.3 \pm 0.9$ ) and that require specific training $(4.4 \pm 0.9$ versus $4.0 \pm 1.0)$ and purchasing or renting of special equipment and services $(4.3 \pm 0.9$ versus $4.1 \pm 1.0)$.

Q2. What level of agreement with the following statements on ITC for Physiotherapy

Q2.1 guarantee the security and privacy

Q2.2 allow in home training for motor rehabilitation

Q2.3 are very expensive

Q2.4 require training

Q2.5 require purchasing or renting of special equipment and services.

\section{Conclusion}

Strategy that promotes tailored information (i.e. that consider digital and health literacy), mainly for elderly population, is needed as a key driver of ICT adoption in physiotherapy. 